The numerical result for $S(x)$ is $7.131 \times 10^{-5}$, compared to the rigorous value of $7.1314 \times 10^{-5}$. Since the error introduced by the quadrature rule is only of the order $e^{-2 x}=e^{-20}$, the main error in this procedure is introduced in the summation process, during which significant figures are lost. Thus, the numerical result obtained here agrees with the analytical result to as many significant figures as were retained during the numerical procedure. However, three of the seven figures to which $\phi(k)$ was originally evaluated were lost during integration.

The same integral has been treated numerically by Simpson's and Filon's methods, and it has been found that in order to obtain accuracy comparable to that obtainable by the method described here the integrand must be evaluated at at least 10 times as many points.

The tables and graph in this paper were prepared by Miss D. M. Keaveney.

H. Hurwitz, JR.

P. F. ZWEIFEL

General Electric Co.

Knolls Atomic Power Laboratory

Schenectady, New York

The Knolls Atomic Power Laboratory is operated by the General Electric Company for the United States Atomic Energy Commission.

1. L. N. G. FILON, "On a quadrature for trigonometric integrals," Royal Soc. Edinburgh, Proc., v. 49, 1928, p. 38-47.

2. YUDELL L. LukE, "On the computation of oscillatory integrals," Cambridge Phil. Soc., Proc., v. 50, 1954, p. 269-277.

3. H. MineUr, Techniques de Calcul Numérique à l'Usage des Mathématiciens, Astronomes, Physiciens et Ingénieurs, Librarie Polytechnique, Paris, 1952; L. V. SPENCER, "Penetration and diffusion of X-Rays: calculation of spatial distributions by semi-asymptotic methods," Phys. Rev., v. 88, 1952, p. 793-803.

4. A. ERdélyi, W. Magnus, F. Oberhettinger, \& F. G. Tricomi, Higher Transcendental Functions, v. 2, McGraw-Hill, Inc., New York, 1953.

5. Thomas Jon I'Anson Bromwich, An Introduction to the Theory of Infinite Series, 2nd Ed., MacMillan \& Co., Ltd., London, 1947.

6. DANIEL SHANKS, "Non-linear transformations of divergent and slowly convergent sequences," J. Math. Phys., v. 34, 1955, p. 1-42. 1928.

7. KonRAD KNOPP, Theory and Application of Infinite Series, Blackie \& Son, Ltd., London,

\title{
Formulas for the Partial Summation of Series
}

1. Introduction. The paper gives a table of the coefficients $A_{m}(n)$ in the "partial" summation formula $S_{n} \doteq \sum_{m=4}^{10} A_{m}(n) S_{m}$. The coefficients $A_{m}(n)$, $m=4(1) 10$, are tabulated exactly in the fractional form $C_{m}(n) / D(n)$ for $n=11(1) 50(5) 100(10) 200(50) 500(100) 1000$. For every $n$, except $47, D(n)$ is the least integer containing no more than ten digits exclusive of final zeros, to permit ready division on a ten-bank calculating machine using $S_{n} \doteq\left[\sum_{m=4}^{10} C_{m}(n) S_{m}\right] / D(n)$. The purpose of $A_{m}(n)$ is the calculation of the sum of $n$ terms of a slowly convergent series, or, more generally, the evaluation of the $n$-th term of a sequence $S_{m}$ which is either slowly convergent or asymptotically characterized by $S_{m} \sim f(m)$ (convergent or divergent), in such a manner that the auxiliary sequence $S_{m} / f(m)$ is slowly convergent. The formula for $S_{n}$ was obtained by Lagrangian extrapolation upon $S_{m}$ considered as a polynomial in $1 / m$, based upon the last 7 values of 
the first 10 terms of the sequence $S_{m}$. Examples of the calculation of $S_{n}$ from the initial values $S_{4}, S_{5}, \cdots, S_{10}$ are given for $S_{m}=\sum_{r=1}^{m} 1 / r^{2}, \sum_{r=1}^{m} 1 / r$, the smallest zero of the $m$-th order Laguerre polynomial, and the $m$-th zero of the Bessel function $J_{0}(x)$, all the results being of high accuracy.

2. Relation to earlier work. In a previous article [1] the writer gave a method, with formulas, for the summation of a slowly convergent series or, more generally speaking, for the evaluation of the limit of a slowly convergent sequence. Those formulas were concerned only with the "complete" sum or limit, i.e., limit $S_{m}$. But in many practical problems we wish to have $S_{m}$ for some larger finite $m \rightarrow \infty$

value of $m$, say $m=n$. For example we may need to estimate the sum of 100 or more terms of a series from the sums of the first few. Then we may have to know the smallest zero of a sequence of polynomials or transcendental functions for some large order from a tabulation of those zeros for only lower orders. The production of this newer table of formulas for "partial" or "incomplete" summation was motivated by the extreme accuracy of the method in the preceding tables of formulas for complete summation, namely, Lagrangian extrapolation for $S_{m}$ considered as a polynomial in $\frac{1}{m}$. Only here, instead of extrapolation for $\frac{1}{m}=0$ $(m=\infty)$ from the last tabulated values up to a fixed $m$, we extrapolate for $\frac{1}{m}=\frac{1}{n}(m=n)$ when we require the value of $S_{n}$. But in employing this method, the sequence $S_{m}$ must always be examined, usually in the roughest way, to see whether it converges slowly as it is, or whether it diverges in some regular manner, in which case it is essential to calculate with some auxiliary sequence obtained by dividing through by some simple function of $m$, often $m$ itself, to insure convergence. Even when the sequence $S_{m}$ does not diverge, knowledge of its asymptotic behavior, say $S_{m} \sim f(m)$ as $m \rightarrow \infty$, enables one to obtain much more accurate results by extrapolating upon the auxiliary sequence $S_{m} / f(m)$ instead of $S_{m}$ (as will be apparent from some of the examples below).

3. Choice of arguments. The earlier article [1], which was devoted only to complete summation, gave sets of multipliers depending upon the number of terms of $S_{m}$ that were available, and also the degree of accuracy that was desired. But since "incomplete" summation requires a separate set of multipliers for every desired $S_{n}$, where $n$ should assume a number of values to be generally useful, to save excessive space it was decided to choose a single number of available values of $S_{m}$, as well as just one degree of approximation, which would serve best in an all-purpose capacity. Careful scrutiny of the examples for complete summation in the previous article [1] showed that the best single all-purpose choice of the total number of available values of $S_{m}$ and the number of end values upon which to base the approximate formulas was 10 and 7 , respectively. Other considerations for the choice of 10 and 7, besides adequacy of the approximation, are:

A. One does not often go beyond the computation of the first 10 terms of a sequence (especially when the calculation may be involved, as in the computation of the roots of transcendental equations) and

B. From the examples in [1] where the results of the 7-point formula were comparable with those of the 4 -point formula, the substantially greater accuracy 
in the former justifies the somewhat larger table of formulas that is needed for 7 instead of 4 points.

4. Range of table. This present table gives the coefficients $A_{m}(n)$, more simply written as $A_{m}$, the $n$ being understood, in the following summation formula:

$$
S_{n} \doteq \sum_{m=4}^{10} A_{m}(n) S_{m}
$$

The coefficients $A_{m}, m=4(1) 10$, are tabulated exactly in the fractional form $C_{m}(n) / D(n)$, more simply written as $C_{m} / D$, the $n$ being understood, for $n=11$ (1) $50(5) 100(10) 200(50) 500(100) 1000$. The left-to-right direction of the columns of $C_{m}$ as $m$ goes from 10 down to 4 was chosen to comply with the natural tendency in extrapolation to start the multiplication with the last or 10 th term of $S_{m}$ and to proceed backwards. The denominator $D(n) \equiv D$ was chosen as the least common denominator of the $A_{m}(n)$, and nearly every $D$ had no more than ten digits exclusive of final zeros, so that use of (1) in a ten-bank desk calculating machine in the convenient form

$$
S_{n} \doteq(1 / D) \sum_{m=4}^{10} C_{m} S_{m}
$$

permits ready division by $D$. In the few cases where the l.c.d. of the $A_{m}(n)$ had more than ten digits for division, both numerators and denominator were multiplied by 2,4 , or 8 , resulting in larger values of $C_{m}$ and $D$, but with no more than ten digits in $D$ exclusive of final zeros, with the sole exception of $n=47$, where division by $D=47^{6}$ is conveniently managed by two successive divisions by $47^{3}=103823$.

At the suggestion of the referee, decimal values of $A_{n}$ have been computed and listed by R. B. Horgan [4].

5. Error terms. In addition to the theoretical error in formula (1) or $\left(1^{\prime}\right)$, the user should always bear in mind the size of the coefficients $A_{m}(n)$ and the fact that in most practical examples the quantities $S_{m}$ are inexact, so that if the error $\sigma_{m}$ in $S_{m}$ satisfies $\left|\sigma_{m}\right| \leq \frac{\leq}{10}$, an upper bound for the error in (1) or (1') besides the theoretical one, is $e \sum_{m=4}^{1}\left|A_{m}\right|$. In many cases the actual error due to in exact values of $S_{m}$ is a small fraction of that upper bound.

6. General formula for $A_{m}(n)$. The arguments $n$ were chosen to fulfill most of the needs in summation or evaluation of sequences. For all other values of $n$ the user may calculate $A_{m}(n)$ from the formula

$$
A_{m}(n)=\frac{m^{6}}{n^{6}} \frac{\prod_{j=4}^{10}(n-j)}{(n-m) \Pi^{\prime}(m-j)},
$$

where $j=m$ is absent from $\Pi^{\prime}$.

7. Method of computation. The computation of this table used (2), the procedure being to first get the seven multipliers $m^{6} / \Pi^{\prime}(m-j), m=4(1) 10$, which are independent of $n$, and to multiply each by the corresponding $\prod_{j=4}^{10}(n-j) /(n-m) n^{6}$. 
For the convenience of the computer, we list those multipliers:

\begin{tabular}{c|c|c|c|c|c|c|c}
$m$ & 10 & 9 & 8 & 7 & 6 & 5 & 4 \\
$\frac{m^{6}}{\Pi^{\prime}(m-j)}$ & $\frac{12500}{9}$ & $\frac{-177147}{40}$ & $\frac{16384}{3}$ & $\frac{-117649}{36}$ & $\frac{972}{1}$ & $\frac{-3125}{24}$ & $\frac{256}{45}$
\end{tabular}

A preliminary functional check upon the $A_{m}(n)$ was performed by using

$$
\sum_{m=4}^{10} A_{m}(n)=1
$$

and a final functional check made use of the formula

$$
\sum_{m=4}^{10} \frac{A_{m}(n)}{m^{6}}=\frac{1}{n^{6}}
$$

The author is grateful to Miss Isabelle Arsham for her assistance in the calculation and checking of the table of $A_{m}(n)$.

8. Illustrations. The following examples are intended to illustrate the wide applicability and great accuracy of this table of $A_{m}(n)$ for many different types of problems:

Example 1: As the most elementary type of problem consider the sum $S_{m}=\sum_{r=1}^{m} 1 / r^{2}$, where the 4 th to 10 th partial sums are, to $10 \mathrm{D}$ :

$$
\begin{aligned}
& S_{4}=1.4236111111 \\
& S_{5}=1.4636111111 \\
& S_{6}=1.4913888889 \\
& S_{7}=1.5117970522 \\
& S_{8}=1.5274220522 \\
& S_{9}=1.5397677312 \\
& S_{10}=1.5497677312
\end{aligned}
$$

Suppose that we want both $S_{15}$ and $S_{24}$, and employ (1), using the table of $A_{m}(n)$ for $n=15$ and $n=24$. The calculated value of $S_{15}$ is 1.5804402838 , which agrees with the true value of $S_{15}=1.5804402834$ to around 4 units in the 10th decimal. The calculated value of $S_{24}$ is 1.6041234045 , which agrees with the true value of $S_{24}=1.6041234036$ to within a unit in the 9 th decimal.

Example 2: Consider the partial sums of the divergent series $S_{m}=\sum_{r=1}^{m} 1 / r$, whose values from $m=4$ up to $m=10$ are, to $10 \mathrm{D}$ :

$$
\begin{aligned}
& S_{4}=2.0833333333 \\
& S_{5}=2.2833333333 \\
& S_{6}=2.4500000000 \\
& S_{7}=2.5928571429 \\
& S_{8}=2.7178571429 \\
& S_{9}=2.8289682540 \\
& S_{10}=2.9289682540
\end{aligned}
$$


Suppose that we wish to calculate $S_{26}$, whose true value is 3.8544197162 . Although $S_{m}$ diverges, the sequence $s_{m}=S_{m}-\log m$ converges slowly to Euler's constant, so that one bases the use of (1) upon $s_{m}, m=4(1) 10$, to calculate $s_{26}$ and to obtain $S_{26}$ from $s_{26}+\log 26$ :

$$
\begin{aligned}
& s_{4}=0.6970389722 \\
& s_{5}=0.6738954209 \\
& s_{6}=0.6582405308 \\
& s_{7}=0.6469469939 \\
& s_{8}=0.6384156012 \\
& s_{9}=0.6317436767 \\
& s_{10}=0.6263831610
\end{aligned}
$$

Employing $s_{m}$ in (1), we find for $n=26, s_{26}=0.5963231116$. Since log 26 $=3.2580965380, S_{26}=s_{26}+\log 26$ is found to be 3.8544196496 , which agrees with the true value to $\frac{2}{3}$ of a unit in the 8 th significant figure.

Example 3: From the known values of the smallest zero of the first ten Laguerre polynomials $L_{m}(x)$, one wishes to calculate the smallest zero of the fifteenth Laguerre polynomial [2]. It is true that the $\nu$-th zero of $L_{m}(x)$, or $x_{\nu}{ }^{(m)}$, converges to zero as $m \rightarrow \infty$ in accordance with the asymptotic approximation $2\left(x_{\nu}(m)\right)^{\frac{1}{2}}=m^{-\frac{1}{2}}(\nu \pi+0(1))$. But a much smoother approximation can be had from the sequence $S_{m}=m x_{1}{ }^{(m)}$; these values from $m=4$ to 10 are, to $9 \mathrm{D}$ :

$$
\begin{aligned}
& S_{4}=1.290190758 \\
& S_{5}=1.317801599 \\
& S_{6}=1.337079625 \\
& S_{7}=1.351305736 \\
& S_{8}=1.362237058 \\
& S_{9}=1.370900049 \\
& S_{10}=1.377934705
\end{aligned}
$$

Use of (1) for $n=15$ yields $S_{15}=1.399617169$, from which $S_{15} / 15=0.09330$ 78113 , agreeing with the true value of $x_{1}{ }^{(15)}=0.0933078120$ to within a unit in the 9 th decimal place.

Example 4: From the first ten tabulated zeros of the Bessel function $J_{0}(x)$ [3], to calculate both the 11 th zero and the $42 \mathrm{nd}$ zero. The sequence $J_{0, m}$ of zeros of $J_{0}(x)$ diverges, but (from the asymptotic formula), $j_{0, m} / m$ converges, and $S_{m}=j_{0, m} / m$ yields a good approximation; the values for $m=4$ to $m=10$, are, to $9 \mathrm{D}$ :

$$
\begin{aligned}
& S_{4}=2.947883610 \\
& S_{5}=2.986183542 \\
& S_{6}=3.011843995 \\
& S_{7}=3.030233804 \\
& S_{8}=3.044058941 \\
& S_{9}=3.054831015 \\
& S_{10}=3.063460647
\end{aligned}
$$


TABLE OF

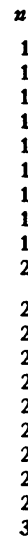

11

12

13

(15

16

$$
C_{10}(n)
$$

7000000

1750000

84000000

1875000

30800000

57750000

1716000000

62562500

5005000000

50050000

1768000000

580125000

27132000000 403750000

10852800000 2331656250

33649000000 1201750000

177100000000

575575000

296010000000

23546250000

52780000000

37110937500 4207320000 1573250000

1107568000000

21014125000

1623160000000 6086850000

2324784000000 2054078125

3262623000000

239898750000

299759200000

327861625000

6096454000000

441190750000

1163580000000

117085237500

720378400000 9019010000

22210148800000

40561950000

57287599600000

546547350000

25963217280000

108832193750

533251118400000

2897025950000

4264761865000

18545034550000

2462578387500

14033106450000

25255333675000

45404248890000

331544548912500

15780722650000

$6626452127 \quad 75000$

113886395350000

7236644062725000 11096398240550000

83140309725000 64449773399550000 352280556473300000 125387677851150000

42152036622850000 275899153284150000 4333642361098050000

20448259635650000 4173696872889950000
$-C_{0}(n)$

11160261

3720087

200884698

4782969

81841914

157837977

4787751969

177324147

14363255907

145083393

5167732284

1707519933

$803347 \quad 47324$ 1201588101

$324428 \quad 78727$ 6997483647

101333929851 3630273471

536473746270 1747909449

900967870530

71816279535
16

161284371885

113600296719

12899667393 4830739641

3405512191752

64696033017

$50031598 \quad 86648$

18782719263

7181259664887 6351251391

10097368902621

$\begin{array}{lll}74 & 30964 & 12747\end{array}$

929275252542

$10171796 \mathbf{6 8 3 2 3}$

18927869617566

1370729296629

3617488594863

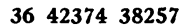

2247096231828

28194539373

$695556538 \quad 78194$

127217409462

$\begin{array}{lll}17990 & 21499 \quad 30594\end{array}$

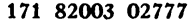

81698199599016

$34 \mathbf{2 7 4 3 0} 51133$

1680577545150504

9136078227063

$\begin{array}{lll}1346 & 41741 & 20579\end{array}$

58600815550893

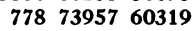

44405031583341

79959181689387

143819281396791

1050611875489836

$\begin{array}{llll}5002 & 48741 \quad 89213\end{array}$

$\begin{array}{lllll}2 & 10126 & 72999 & 12417\end{array}$

361242124646949

22979348857127124

35260896565634607

264327581978739

$\begin{array}{llll}204 & 98191 & 74911 & 47797\end{array}$

1120750826307481512

399002626355163831

134180498395752039

878471584299269631 $\begin{array}{llll}13800 & 98178 & 29522 & 42307\end{array}$

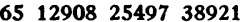
13295012533363791153
$C_{8}(n)$

9175040

3440640

198180864

4915200

86507520

170311680

5248122880

196804608

16102195200

164003840

5882511360

1955266560

92462383104 1389158400

37654364160 8149729280

118385541120 4252925952

630063104000

2057502720

1062744883200

84871987200

190936252416

134701056000

5318384640 $\mathbf{5 7 4 4 3} \mathbf{9 4 2 4 0}$

$\begin{array}{lll}405 & 47804 & 77440\end{array}$

$7 \mathbf{7 1 2 2 1} 74976$

5970749030400

22438563840

8587397038080 7601848320

12096061046784

890913587200

1114987560960

1221351505920

22742887301120

1648090988544

$43521933 \quad 31200$

438474178560

2712105123840

34100183040

84269512785920

154351042560

218539708907520

2089414082560

99439796551680

417507901440

2048625758699520

11143926743040

16440907857920

71619863715840

9524510392320

54344352890880

97909308456960

$\begin{array}{llll}17618 & 76032 & 71680\end{array}$

1287591321927680

61330787696640

2576993736130560

443154741585920

28220471720017920

43333958419906560

325009181573120

252133826986229760

1378955533245480960

491040165764628480

$\begin{array}{llll}165 & 18859 & 10014 & 77120\end{array}$

1081744124362260480 16997543391194562560

80225546481991680 16378555903977738240

$-C_{7}(n)$

$41 \quad 17715$

1647086

98825160

2521050

45294865

90589730

2826399576

107060590

8832498675

90589730

3268625360 
$A_{m}(n) \equiv C_{m}(n) / D(n)$

$$
C_{6}(n)
$$

979776

408240

25194240

656100

11975040

24249456

764225280

29189160

2424945600

25019280

907365888

304496010

14520280320

219769200

5996229120

1305429048

19063408320

688117680

102386592000

335675340

174014096256

13943437200

31465324800

22261473000

2538319680

954220176

675104855040

12868209585

998261107200

3758665680

1441036624896 1277800920

2036469314880

150218031600

188267224320

206505611676

3850294331520

279356940720

738569145600

74491756920

$4629945 \quad 48480$ 5844318480 14489750499840 26612695395

$377679708 \quad 80640$

$\begin{array}{lll}36 & 18202 & 54320\end{array}$

17250093020160

$725382 \quad 11880$

$3564178576 \quad 35840$

1941179975280

2869856676540

12523169015280

1667816960040

$95277671 \quad 51760$

17183729032470

30950366438160

226368914254560

10790096320080

453664199062620

78058909459440

4981468505761440

$7 \quad 6600475168 \quad 47920$

57508345586340

$\begin{array}{lllll}44 & 64661 & 54117 & 18320\end{array}$

244318943339190720

87040775524293360

$293010302575 \quad 41840$ 191972380071645360 3017
577397094179920

14246480895791760 $2909 \begin{array}{lll}16581 & 42133 \quad 49680\end{array}$

$$
\begin{aligned}
& \begin{aligned}
&-C_{b}(n) \\
& 109375 \\
& 46875
\end{aligned} \\
& 46875 \\
& \begin{array}{l}
53125 \\
78125
\end{array} \\
& 1443750 \\
& 2953125 \\
& 93843750 \\
& 3609375 \\
& 301640625 \\
& 3128125
\end{aligned}
$$

664589760862500

1022655346321875

7681441659375

5965683139040625

32655220122525000

11636356467246875

3918545421346875 $\begin{array}{llll}25 & 67946 & 25583 & 71875\end{array}$ $40372375 \quad 76966 \quad 34375$

1906314707653125 389317830165928125
28918335688704 $4452 \quad 95462 \quad 36672$

$3346373 \quad 43744$

$\begin{array}{llll}25998 & 64798 & 46912\end{array}$

1423529394839552

507375171278976

170916607020544

1120340837611776

17616800625295872

83195204917616

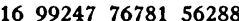

$$
D(n)
$$

1771561

186624

4826809

67228

759375

1048576

24137569

708588

47045881

400000

12252303

3543122

148035889

1990656

48828125

9653618

129140163

4302592

594823321

1822500

887503681

67108864

143496441

96550276

10504375

3779136

2565726409

47045881

3518743761

12800000

4750104241

4084101

6321363049

453519616

553584375

592143556

10779215329

764411904

1977326743

195312500

1107225625

12960000

30167556250

52521875

71191406250 655360000

30171961250

123018750

$58 \quad 8073512500$

3125000000

4428902500

18662400000

2413404500

13445600000

$237304 \quad 68750$

41943040000

301719612500

14171760000

$\begin{array}{llll}58 & 80735 \quad 12500\end{array}$

100000000000

6103515625000 9112500000000

66992187500

276792187500000

97656250000000

32400000000000

210087500000000

3276800000000000

15377343750000

3125000000000000 
Use of the above values of $S_{m}$ in (1) for $n=42$ gave $S_{42}=3.122914703$, from which $42 \times S_{42}=131.1624175$, agreeing with the true value of 131.1624463 to within 3 units in the 8 th significant figure. But the same formula (1) for the very next or 11 th zero was so accurate as to give an answer of 33.77582019 , which agrees with the true value of 33.77582021 to 2 units in the 10 th significant figure.

Convair

\section{Herbert E. Salzer}

San Diego 12, California

The work reported was done while the author worked at the Diamond Ordnance Fuze Laboratories, Washington, D. C.

1. H. E. SALZER, "A simple method for summing certain slowly convergent series," J. Math. Phys., v. 33, 1955, p. 356-359.

2. H. E. SALZER \& R. ZUCKER, "Table of the zeros and weight factors of the first fifteen Laguerre polynomials," Amer. Math. Soc., Bull., v. 55, 1949, p. 1004-1012.

3. British Association for the Advancement of Science, Mathematical Tables, v. VI, Bessel Functions, Part I, Cambridge Univ. Press, 1950, p. 171.

4. R. B. HoRGAN, "Table of coefficients for the partial summation of series" [see note below, this issue].

\section{TECHNICAL NOTES AND SHORT PAPERS}

\section{Table of Coefficients for the Partial Summation of Series}

This note supplies a table of the coefficients $A_{m}(n)$ as described by Herbert E. Salzer [1]. The computation of the table was undertaken at the suggestion of the referee of Dr. Salzer's paper and the Chairman of the Editorial Committee. Responsibility for its correctness does not fall upon Dr. Salzer.

For $m=4(1) 10$ and $n=11(1) 50(5) 100(10) 200(50) 500(100) 1000$, the table lists

$$
A_{m}(n)=\frac{m^{6} \prod_{j=4}^{10}(n-j)}{n^{6}(n-m) \Pi^{\prime}(m-j)}
$$

to fifteen decimals. ( $j=m$ is absent from $\Pi^{\prime}$.)

The computation was performed on the SWAC machine by means of double precision routines. The seven values of

$$
\frac{m^{6}}{\Pi^{\prime}(m-j)}
$$

were stored as constants in the computing routine. For each $n$, the seven values of $\frac{\prod_{j=4}^{10}(n-j)}{n^{6}(n-m)}$ were obtained and then multiplied by (2), resulting in (1).

The results were converted to 25-decimal numbers and punched on cards by SWAC. They were listed on an International Business Machines Corporation 